\title{
Measurement of light mesons at RHIC by the PHENIX experiment
}

\author{
M. Naglis ${ }^{\mathrm{a}}$ \\ for the PHENIX Collaboration \\ Weizmann Institute of Science, Rehovot 76100, Israel
}

Received: 13 September 2008 / Revised: 16 December 2008 / Published online: 5 February 2009

(C) Springer-Verlag / Società Italiana di Fisica 2009

\begin{abstract}
The PHENIX experiment at RHIC has measured a variety of light neutral mesons $\left(\pi^{0}, \mathrm{~K}_{S}^{0}, \eta, \omega, \eta^{\prime}, \phi\right)$ via multi-particle decay channels over a wide range of transverse momentum. A review of the recent results on the production rates of light mesons in $\mathrm{p}+\mathrm{p}$ and their nuclear modification factors in $\mathrm{d}+\mathrm{Au}, \mathrm{Cu}+\mathrm{Cu}$ and $\mathrm{Au}+\mathrm{Au}$ collisions at different energies is presented.
\end{abstract}

\section{Introduction}

The suppression of high- $p_{T}$ light mesons in ultra-relativistic heavy ion collisions, compared to expectations from scaled $\mathrm{p}+\mathrm{p}$ results, is one of the most interesting findings at RHIC. Particles with high transverse momentum are believed to be produced by fragmentation of hard-scattered partons in the earliest stage of the collision. In nucleus-nucleus collisions, the scattered hard partons lose their energy while traversing the subsequently produced hot and dense matter leading to the phenomenon of jet-quenching. Medium-induced effects on high- $p_{T}$ particle production can be quantified with the nuclear modification factor:

$R_{A A}\left(p_{T}\right)=\frac{d^{2} N_{A A} / d y d p_{T}}{\left(\left\langle N_{c o l l}\right\rangle / \sigma_{p p}^{\text {inel }}\right) \times d^{2} \sigma_{p p} / d y d p_{T}}$,

where $d^{2} N_{A A} / d y d p_{T}$ is the differential yield per event in nucleus-nucleus collisions, $\left\langle N_{\text {coll }}\right\rangle$ is the number of binary nucleon-nucleon collisions averaged over the impact parameter range of the corresponding centrality, and $\sigma_{p p}^{\text {inel }}$ and $d^{2} \sigma_{p p} / d y d p_{T}$ are the total and differential cross sections for inelastic $\mathrm{p}+\mathrm{p}$ collisions, respectively. In the absence of medium-induced effects, the yield of high- $p_{T}$ particles is expected to scale with $\left\langle N_{\text {coll }}\right\rangle$, resulting in $\mathrm{R}_{A A}=1$ at high- $p_{T}$. Contrary to the suppression of mesons, baryons

a e-mail: maxim.naglis@weizmann.ac.il are found to be enhanced in central $\mathrm{Au}+\mathrm{Au}$ collisions at RHIC at intermediate transverse momenta $\left(1.5<p_{T}<\right.$ $4.5 \mathrm{GeV} / c$ ) [1]. The difference in the behavior of mesons and baryons is still not well understood.

Precise knowledge of meson and baryon yields and their ratios as a function of transverse momentum and in different collision systems, provides a mean to investigate the dependence of hadron formation on particle mass and quarkflavor composition, and the mechanisms of jet production and quenching. The PHENIX experiment excels in measuring light neutral mesons via multi-particle decay channels. This paper presents a review of the light meson data obtained in $\mathrm{p}+\mathrm{p}$ and heavy-ion collisions at different energies.

\section{Experimental setup and data analysis}

The PHENIX detector [2] at RHIC (Relativistic Heavy Ion Collider) has been designed to measure a broad variety of signals from heavy ion collisions. PHENIX is capable of measuring electrons, photons and hadrons in a large dynamic range. The tracking of charged particles and measurements of their momentum are accomplished by the highresolution multi-wire proportional Drift Chambers (DC) and the first layer of Pad Chambers (PC1). The typical value of the momentum resolution is $\sigma\left(p_{T}\right) / p_{T} \approx 1.0 \% p_{T} \oplus 1.1 \%$. The energy of photons and electrons is measured by highly segmented lead-glass $(\mathrm{PbGl})$ and lead-scintillator $(\mathrm{PbSc})$ Electro Magnetic Calorimeters (EMCal) which have an energy resolution of $\sigma_{P b G l}(E) / E \approx 5.9 \% / \sqrt{E} \oplus 0.8 \%$ and $\sigma_{P b S c}(E) / E \approx 8.1 \% / \sqrt{E} \oplus 2.1 \%$, respectively. Charged hadron identification is based on the particle mass calculated from the momentum and the time-of-flight information derived from the TOF or EMCal subsystems and the Beam Beam Counters (BBC's). The TOF subsystem with a time resolution $\sigma \simeq 120 \mathrm{ps}$ and the PbSc part of the EMCal with $\sigma \simeq 500$ ps allow to achieve reliable pion-kaon separation 
in the $p_{T}$ ranges $0.3-2.5 \mathrm{GeV} / c$ and $0.3-1.0 \mathrm{GeV} / c$, respectively. The Zero Degree Calorimeters (ZDC's) and BBC's are dedicated subsystems that determine the collision vertex and event centrality and also provide the minimum bias (MB) interaction trigger. The results of data presented in this paper are based on data samples accumulated during $\mathrm{p}+\mathrm{p}, \mathrm{d}+\mathrm{Au}, \mathrm{Cu}+\mathrm{Cu}$ and $\mathrm{Au}+\mathrm{Au}$ collisions at $\sqrt{s_{N N}}=$ $200 \mathrm{GeV}, \mathrm{p}+\mathrm{p}$ and $\mathrm{Cu}+\mathrm{Cu}$ collisions at $\sqrt{s_{N N}}=62.4 \mathrm{GeV}$, and $\mathrm{Cu}+\mathrm{Cu}$ collisions at $\sqrt{s_{N N}}=22.4 \mathrm{GeV}$. The MB trigger used for $\mathrm{p}+\mathrm{p}, \mathrm{d}+\mathrm{Au}$ and $\mathrm{Cu}+\mathrm{Cu}$ collisions requires a coincidence between the BBC's with at least one hit in each $\mathrm{BBC}$ arm. For $\mathrm{Au}+\mathrm{Au}$ collisions the $\mathrm{MB}$ trigger requires a coincidence between the BBC's and ZDC's with at least two hits in each BBC arm, at least one neutron detected in each ZDC arm. Besides, PHENIX utilizes a hardware high$p_{T}$ photon/electron trigger (ERT) based on the analog sum of the signals of adjacent $4 \times 4$ EMCal towers in coinci- dence with the MB trigger condition. All events used in the analysis are required to have the collision vertex position along the beam axis within $30 \mathrm{~cm}$ of the geometrical center of PHENIX.

A summary of the decay channels studied and data samples analyzed is given in Table 2.1. The procedure used to reconstruct the invariant mass spectra of $\pi^{0}, \mathrm{~K}_{S}^{0}, \eta, \eta^{\prime}, \omega$ mesons is as follows. All photon clusters measured in the EMCal in a given event are paired to form the invariant mass distribution. Pairs with invariant mass within two standard deviations from the $\pi^{0}(\eta)$ mass are considered as $\pi^{0}(\eta)$ candidates. Raw $\pi^{0}(\eta)$ yields are determined by subtracting the yield of combinatorial pairs estimated by pairing photon clusters from different events. Then, the selected $\pi^{0}$ $(\eta)$ candidates are combined between themselves, or with other photon clusters, or with charged tracks, according to the decay modes listed in Table 2.1. The invariant mass

Table 2.1 Summary of analyzed decay channels and data samples. In $\mathrm{Au}+\mathrm{Au}(\mathrm{p}+\mathrm{p})$ collisions, the measurements of the $\phi$ meson were performed using two different analysis techniques referred to in text as "two kaons PID" and "no PID" (“one kaon PID" and "no PID”), respectively

\begin{tabular}{|c|c|c|c|c|c|c|}
\hline Data sample & $\sqrt{s_{N N}}(\mathrm{GeV})$ & $\int L d t$ & Trigger & Decay mode & $p_{T}(\mathrm{GeV} / c)$ & Reference \\
\hline Run-2 $\mathrm{Au}+\mathrm{Au}$ & 200 & $24 \mu \mathrm{b}^{-1}$ & MB & $\eta \rightarrow \gamma \gamma$ & $2.25-9.5$ & {$[6]$} \\
\hline \multirow[t]{5}{*}{ Run-3 p + p } & \multirow[t]{5}{*}{200} & \multirow[t]{5}{*}{$0.35 \mathrm{pb}^{-1}$} & ERT & $\mathrm{K}_{S}^{0} \rightarrow \pi^{0} \pi^{0}$ & $2.5-6.5$ & [7] \\
\hline & & & $\mathrm{MB}$ & $\eta \rightarrow \gamma \gamma$ & $2.75-11.0$ & {$[6]$} \\
\hline & & & ERT & $\eta \rightarrow \pi^{0} \pi^{+} \pi^{-}$ & $3.0-8.0$ & [6] \\
\hline & & & ERT & $\omega \rightarrow \pi^{0} \pi^{+} \pi^{-}$ & $2.75-9.25$ & {$[5]$} \\
\hline & & & ERT & $\omega \rightarrow \pi^{0} \gamma$ & $2.5-6.5$ & [5] \\
\hline \multirow[t]{6}{*}{ Run-3 d $+A u$} & \multirow[t]{6}{*}{200} & \multirow[t]{6}{*}{$2.74 \mathrm{nb}^{-1}$} & ERT & $\mathrm{K}_{S}^{0} \rightarrow \pi^{0} \pi^{0}$ & $3.5-8.5$ & [7] \\
\hline & & & MB & $\eta \rightarrow \gamma \gamma$ & $2.25-11.0$ & {$[6]$} \\
\hline & & & ERT & $\eta \rightarrow \pi^{0} \pi^{+} \pi^{-}$ & $5.0-8.0$ & [6] \\
\hline & & & ERT & $\omega \rightarrow \pi^{0} \pi^{+} \pi^{-}$ & $3.5-9.0$ & {$[5]$} \\
\hline & & & ERT & $\omega \rightarrow \pi^{0} \gamma$ & $3.0-7.0$ & [5] \\
\hline & & & $\mathrm{MB}$ & $\phi \rightarrow \mathrm{K}^{+} \mathrm{K}^{-}$ & $1.45-5.1$ & [9] \\
\hline \multirow[t]{4}{*}{ Run- $4 \mathrm{Au}+\mathrm{Au}$} & \multirow[t]{4}{*}{200} & \multirow[t]{4}{*}{$241 \mu b^{-1}$} & $\mathrm{MB}$ & $\pi^{0} \rightarrow \gamma \gamma$ & $1.25-19.0$ & [15] \\
\hline & & & MB & $\omega \rightarrow \pi^{0} \gamma$ & $4.0-9.0$ & [7] \\
\hline & & & MB & $\phi \rightarrow \mathrm{K}^{+} \mathrm{K}^{-}$ & $0.8-4.0$ & {$[8]$} \\
\hline & & & MB & $\phi \rightarrow \mathrm{K}^{+} \mathrm{K}^{-}$ & $2.45-7.0$ & - \\
\hline \multirow[t]{7}{*}{ Run-5 $p+p$} & \multirow[t]{7}{*}{200} & \multirow[t]{7}{*}{$3.78 \mathrm{pb}^{-1}$} & MB/ERT & $\pi^{0} \rightarrow \gamma \gamma$ & $0.625-19.0$ & {$[14]$} \\
\hline & & & ERT & $\mathrm{K}_{S}^{0} \rightarrow \pi^{0} \pi^{0}$ & $2.25-10.0$ & [7] \\
\hline & & & ERT & $\omega \rightarrow \pi^{0} \pi^{+} \pi^{-}$ & $2.25-13.0$ & [7] \\
\hline & & & ERT & $\omega \rightarrow \pi^{0} \gamma$ & $2.5-11.0$ & [7] \\
\hline & & & ERT & $\eta^{\prime} \rightarrow \eta \pi^{+} \pi^{-}$ & $3.25-10.75$ & - \\
\hline & & & MB & $\phi \rightarrow \mathrm{K}^{+} \mathrm{K}^{-}$ & $0.9-4.5$ & - \\
\hline & & & MB & $\phi \rightarrow \mathrm{K}^{+} \mathrm{K}^{-}$ & $1.3-7.0$ & - \\
\hline \multirow[t]{3}{*}{ Run-5 $\mathrm{Cu}+\mathrm{Cu}$} & 200 & $3.06 \mathrm{nb}^{-1}$ & MB/ERT & $\pi^{0} \rightarrow \gamma \gamma$ & $1.25 .0-11.0$ & [12] \\
\hline & 62.4 & $0.19 \mathrm{nb}^{-1}$ & $\mathrm{MB}$ & $\pi^{0} \rightarrow \gamma \gamma$ & $1.25-7.5$ & [12] \\
\hline & 22.4 & $2.7 \mu \mathrm{b}^{-1}$ & MB & $\pi^{0} \rightarrow \gamma \gamma$ & $1.25-4.75$ & [12] \\
\hline Run- $6 p+p$ & 62.4 & $0.1 \mathrm{pb}^{-1}$ & MB/ERT & $\pi^{0} \rightarrow \gamma \gamma$ & $0.625-6.75$ & [12] \\
\hline
\end{tabular}


spectra of the $\phi$ meson are constructed using three different techniques. The first does not require track identification in the final state and assumes that all tracks are kaons ("no PID"). The second requires identification of only one kaon in the TOF subsystem ("one kaon PID"). In the third technique both kaons are identified in the TOF or EMCal subsystems ("two kaons PID"). Kaon candidates with opposite charge are combined to form the invariant mass distribution. Mass distributions obtained by the procedures described above contain both the signal and an inherent combinatorial background. Meson raw yields were extracted by simultaneously fitting the signal and background, or by integrating the spectra in the vicinity of the meson masses after subtraction of the combinatorial background, estimated using an event-mixing technique. Representative examples of the $\mathrm{K}^{+} \mathrm{K}^{-}$invariant mass distributions obtained using "no PID", "one kaon PID" techniques in $\mathrm{p}+\mathrm{p}$ collisions and using "no PID" technique in 0-10\% most central $\mathrm{Au}+\mathrm{Au}$ collisions, and an example of the $\eta \pi^{+} \pi^{-}$invariant mass distribution are shown in Fig. 2.1. Solid lines are fits to a sum of a Gaussian convoluted Breit-Wigner function to account

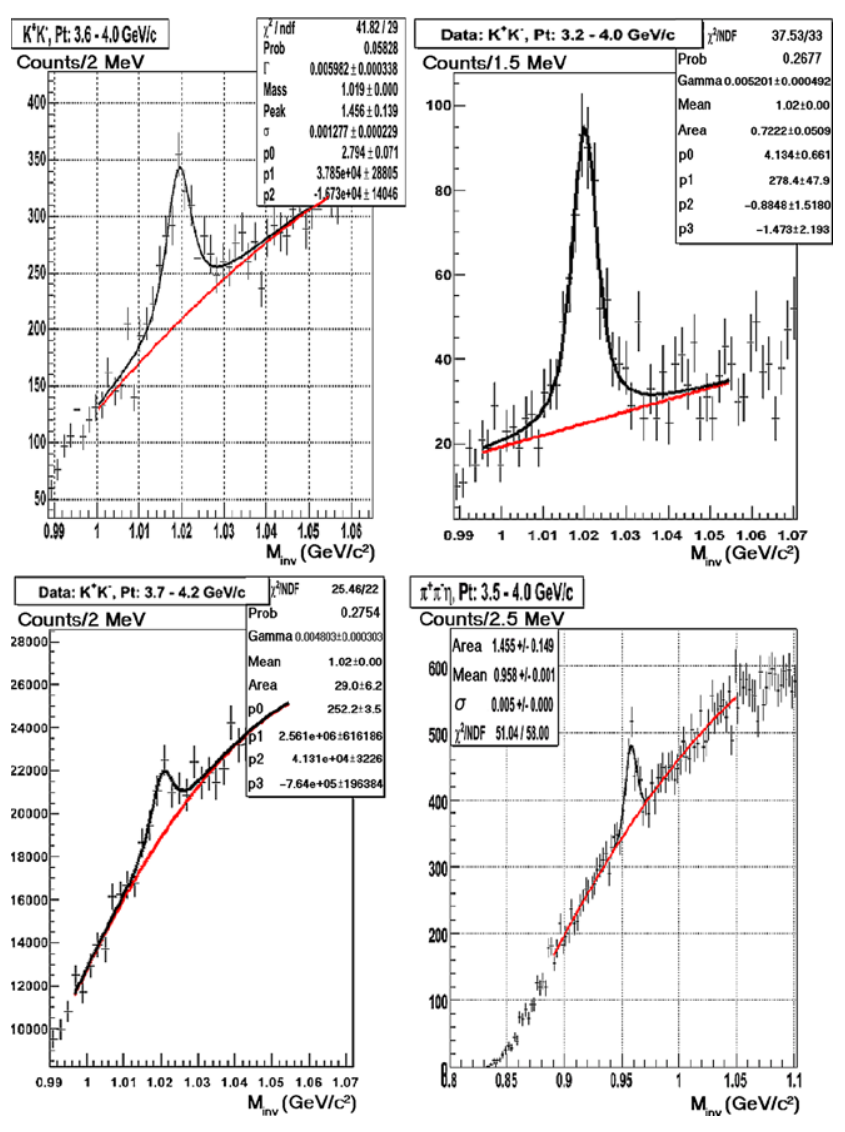

Fig. 2.1 Invariant mass distributions of the $\phi$ mesons reconstructed in the $\mathrm{K}^{+} \mathrm{K}^{-}$decay channel using "no PID" (top left), "one kaon PID" (top right) techniques in $\mathrm{p}+\mathrm{p}$ collisions and using "no PID" (bottom left) technique in $0-10 \%$ most central $\mathrm{Au}+\mathrm{Au}$ collisions. Invariant mass distribution of the $\eta^{\prime}$ mesons reconstructed in the $\eta \pi^{+} \pi^{-}$decay channel (bottom right) in $\mathrm{p}+\mathrm{p}$ collisions for the $\phi$ signal, or a pure Gaussian for the $\eta^{\prime}$ signal, and a polynomial background function. Corrections to the raw yields for the limited detector acceptance and resolution, reconstruction and trigger efficiency, multiplicity effects and various analysis cuts are determined from the full singleparticle Monte Carlo simulation and analysis of the data.

\section{Results}

\subsection{Particle spectra}

The study of inclusive particle production in nucleonnucleon collisions is a rich source of information on the fragmentation properties of partons in perturbative QCD and hadronization mechanisms. The PHENIX experiment has measured a variety of mesons in $\mathrm{p}+\mathrm{p}$ collisions at $\sqrt{s}=$ $200 \mathrm{GeV}$. These data in comparison to existing leadingand next-to-leading order perturbative QCD calculation can be used to constrain the model parameters. A compilation of published and preliminary results on meson production in $\mathrm{p}+\mathrm{p}$ collisions is presented in Fig. 3.1. The different symbols for a given meson correspond to different decay channels or different analysis techniques. The third set of points from the bottom represents the first measurement of $\eta^{\prime} \rightarrow \eta \pi^{+} \pi^{-}$at RHIC at high- $p_{T}$. The solid lines shown in the figure are just to guide the eye.

The $\phi$ meson invariant $p_{T}$ spectra measured in $\mathrm{p}+\mathrm{p}$, $\mathrm{d}+\mathrm{Au}$ and $\mathrm{Au}+\mathrm{Au}$ collisions at $\sqrt{s_{N N}}=200 \mathrm{GeV}$ are

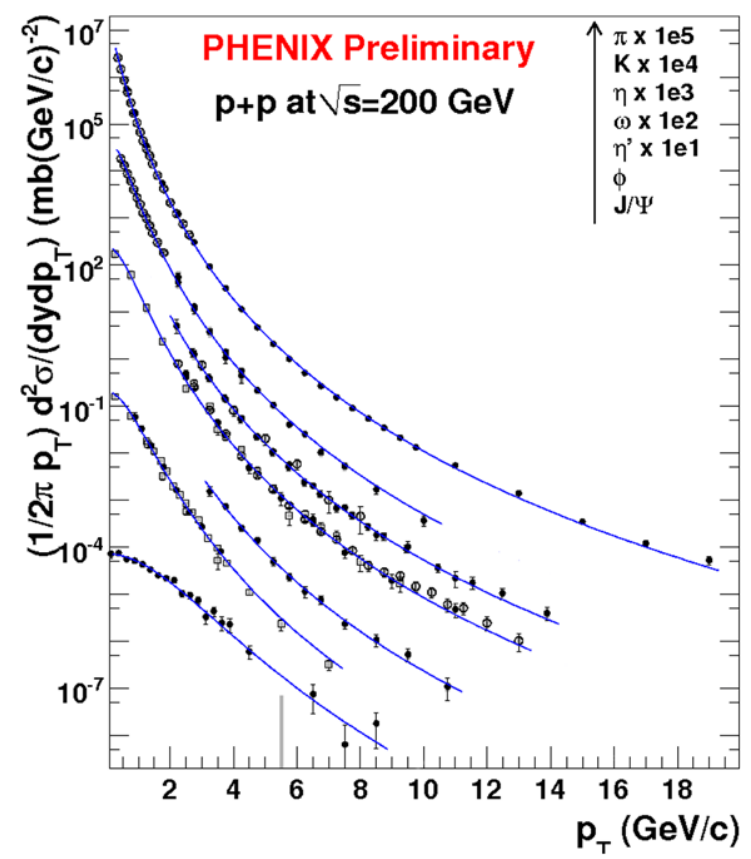

Fig. 3.1 Compilation of PHENIX results on meson production in $\mathrm{p}+\mathrm{p}$ collisions at $\sqrt{s}=200 \mathrm{GeV}$. The solid lines are just to guide the eye. Charged kaon and pion data are taken from [3], $J / \Psi$ data are taken from [4] 
Fig. 3.2 Invariant $p_{T}$ spectra of $\phi$ meson measured in $\mathrm{p}+\mathrm{p}$, $\mathrm{d}+\mathrm{Au}$ and $\mathrm{Au}+\mathrm{Au}$ collisions at $\sqrt{s_{N N}}=200 \mathrm{GeV}$

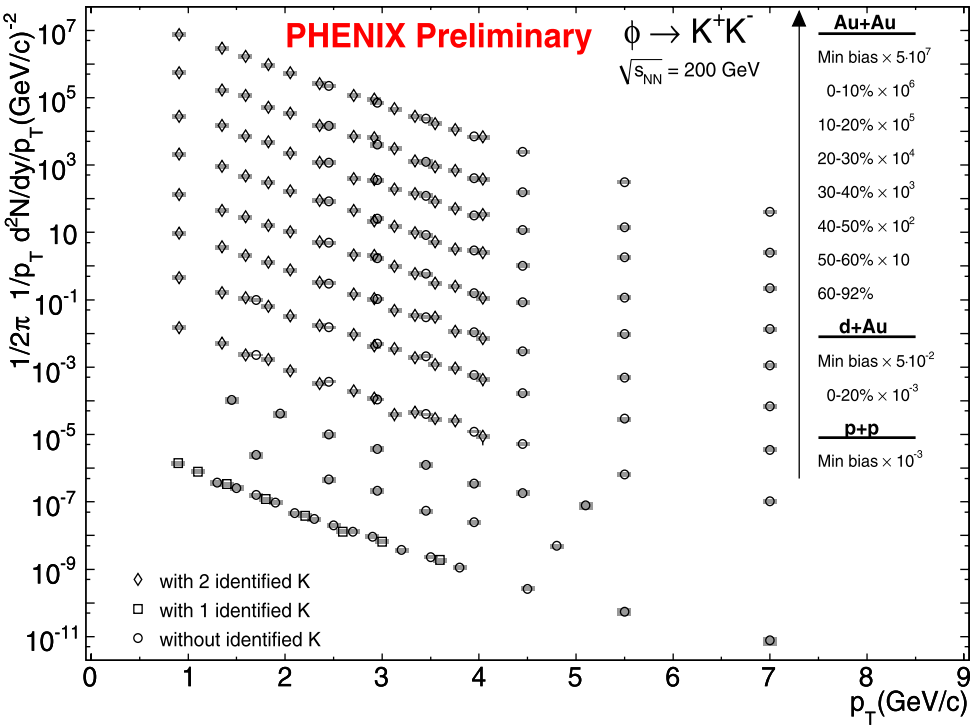

Table 3.1 Results of the fits to a constant for $\eta / \pi^{0}, \mathrm{~K}_{S}^{0} / \pi^{0}$ and $\omega / \pi^{0}$ ratios in $\mathrm{p}+\mathrm{p}$ and $\mathrm{d}+\mathrm{Au}$ collisions at $\sqrt{s_{N N}}=200 \mathrm{GeV}$

\begin{tabular}{llll}
\hline System & $\eta / \pi^{0}$ & $\mathrm{~K}_{S}^{0} / \pi^{0}$ & $\omega / \pi^{0}$ \\
\hline $\mathrm{p}+\mathrm{p}$ & $0.48 \pm 0.02$ (stat) \pm 0.02 (syst) & $0.45 \pm 0.01$ (stat) \pm 0.05 (syst) & $0.81 \pm 0.03$ (stat) \pm 0.07 (syst) \\
$\mathrm{d}+\mathrm{Au}$ & $0.47 \pm 0.02$ (stat) \pm 0.02 (syst) & $0.58 \pm 0.06$ (stat) \pm 0.05 (syst) & $0.94 \pm 0.08$ (stat) \pm 0.12 (syst) \\
\hline
\end{tabular}

shown in Fig. 3.2. As compared to the previously reported $\mathrm{Au}+\mathrm{Au}$ results [8] obtained with the "two kaons PID" approach, the new "no PID" analysis extends the range of the measurements to higher $p_{T}$ up to $7 \mathrm{GeV} / c$. In turn, the new "one kaon PID" analysis of $p+p$ data extends the range of the "no PID" measurements reported in [9] to lower $p_{T}$ down to $0.9 \mathrm{GeV} / c$. In spite of the very different sources of systematic uncertainties, the results of the "no PID" analysis are in good agreement with the results of "one kaon PID" and "two kaons PID" in $\mathrm{p}+\mathrm{p}$ and $\mathrm{Au}+\mathrm{Au}$ collisions, respectively. The combined $\mathrm{p}+\mathrm{p}$ results of the "one kaon PID" and "no PID" analyses constitute a new $p+p$ reference for $\phi$ meson, which supersedes the one used previously [8] having both a wider $p_{T}$ range and smaller error bars.

The details of the $\mathrm{K}_{S}^{0}, \eta$ and $\omega$ measurements in $\mathrm{p}+\mathrm{p}$, $\mathrm{d}+\mathrm{Au}$ and $\mathrm{Au}+\mathrm{Au}$ collisions at $\sqrt{s_{N N}}=200 \mathrm{GeV}$ can be found elsewhere [5-7]. Good agreement was obtained in the measurements of the same meson via two different decay channels, $\omega \rightarrow \pi^{0} \pi^{+} \pi^{-}$or $\omega \rightarrow \pi^{0} \gamma$ and $\eta \rightarrow \pi^{0} \pi^{+} \pi^{-}$or $\eta \rightarrow \gamma \gamma$. Analyses of MB and ERT data samples collected in Run-3 and Run-5 gave consistent results.

\subsection{Particle ratios}

Non-identical particle ratios measured at high- $p_{T}$ are useful to assess the particle composition of jet fragmentation.
In $\mathrm{p}+\mathrm{p}$ collisions, meson-to-meson ratios $\left(\eta / \pi^{0}, K / \pi^{0}\right.$, $\left.\eta^{\prime} / \pi^{0}, \omega / \pi^{0}, \phi / \pi^{0}, \phi / K\right)$ derived using a parameterization of the $\pi^{0}$ invariant $p_{T}$ spectra [10] exhibit a universal flat behavior above $p_{T}=2.5 \mathrm{GeV} / c$ [9] indicating that the measured transverse momentum spectra for all light mesons have the same slope at high- $p_{T}$. Similarly to $\mathrm{p}+\mathrm{p}$ results, $\eta / \pi^{0}, \mathrm{~K}_{S}^{0} / \pi^{0}$ and $\omega / \pi^{0}$ ratios in $\mathrm{d}+\mathrm{Au}$ collisions are also found to be flat in the same $p_{T}$ range. Results of the fits to a constant performed in the $p_{T}$ region above $2.5 \mathrm{GeV} / c$ for $\eta / \pi^{0}, \mathrm{~K}_{S}^{0} / \pi^{0}$ and $\omega / \pi^{0}$ ratios are presented in Table 3.1. As one can see, the results obtained in $p+p$ and $\mathrm{d}+\mathrm{Au}$ collisions are consistent within errors for the same ratio, indicating that the production of light mesons at $p_{T}>2.5 \mathrm{GeV} / c$ is not affected by cold nuclear matter.

\subsection{Nuclear modification factors}

Using the new $\mathrm{p}+\mathrm{p}$ reference, $\phi$ mesons's $\mathrm{R}_{A A}$ has been derived in central $\mathrm{Au}+\mathrm{Au}$ collisions in the $p_{T}$ range of 2.45-7 GeV/c. There is an ongoing work to extend this $p_{T}$ range towards low- $p_{T}$. Figure 3.3 shows a comparison of the $\mathrm{R}_{A A}$ for $\phi$ mesons, other mesons $\left(\pi^{0}, \eta, \mathrm{K}, \omega\right)$, protons and direct photons. Neutral pions and $\eta$ mesons follow the same suppression pattern over the entire $p_{T}$ range. The $R_{A A}$ of $\phi$ mesons exhibits less suppression than $\eta$ and $\pi^{0}$ in the $p_{T}$ range of $2.45<p_{T}<4.5 \mathrm{GeV} / c$. At 


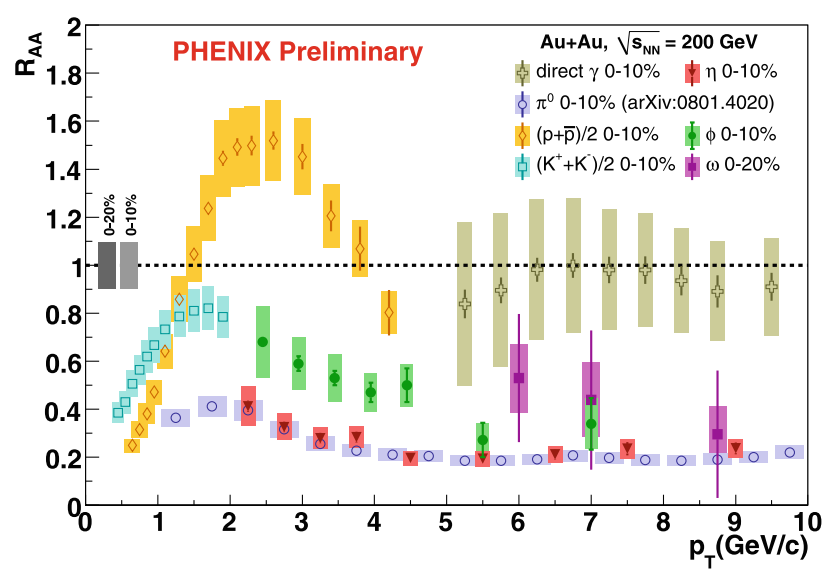

Fig. 3.3 Nuclear modification factor $R_{A A}$ in central $\mathrm{Au}+\mathrm{Au}$ collisions as a function of $p_{T}$ for $\pi^{0},\left(\mathrm{~K}^{+}+\mathrm{K}^{-}\right) / 2, \eta, \omega,(\mathrm{p}+\overline{\mathrm{p}}) / 2, \phi$ and direct $\gamma$. Direct $\gamma$ data are taken from [11]

higher $p_{T}(>5 \mathrm{GeV} / c)$ the amount of suppression of $\phi$ and $\omega$ could be similar to that of $\pi^{0}$ and $\eta$ mesons. A more conclusive statement is not possible due to the large errors of the measurements. The similarity between the suppression patterns of different mesons at high- $p_{T}$ favors the production of these mesons via jet fragmentation outside the hot and dense medium created in the collision. It is not clear whether the $R_{A A}$ of charged kaons follow or not the trend of $\phi$ mesons, since the present measurements have no overlap in $p_{T}$. Interestingly, that $R_{A A}$ of protons which are known to be enhanced in the intermediate $p_{T}$ range [1], reach a value below unity at $p_{T} \approx 4.2 \mathrm{GeV} / c$. Further investigations are required for understanding the influence of quark flavor composition on the suppression pattern.

\subsection{Onset of jet quenching}

In contrast to RHIC results at $\sqrt{s_{N N}}=130 \mathrm{GeV}$ and $200 \mathrm{GeV}$, no suppression of high- $p_{T}$ hadrons has been observed at SPS energies. This implies that there must be an intermediate value of collision energy at which the onset of high- $p_{T}$ suppression happens. The results of a beam energy scan performed at RHIC with $\mathrm{Cu}+\mathrm{Cu}$ collisions at $\sqrt{s_{N N}}=22.4 \mathrm{GeV}, 62.4 \mathrm{GeV}$ and $200 \mathrm{GeV}$, shown in Fig. 3.4, indicate that parton energy loss starts to dominate over Cronin enhancement at a center of mass energy somewhere between 22.4 and $62.4 \mathrm{GeV}$ per nucleon pair [12]. Numerical calculations based on a parton energy loss model [13] describe well the suppression of $\pi^{0}$ mesons above $p_{T}=3 \mathrm{GeV} / c$ at $62.4 \mathrm{GeV}$ and $200 \mathrm{GeV}$. Measurements at $22.4 \mathrm{GeV}$ favor the calculations carried out without taking into account parton energy loss.

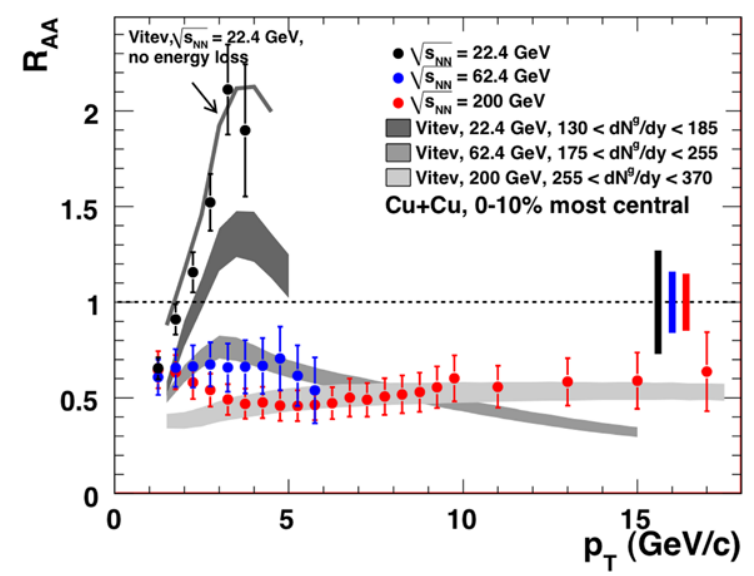

Fig. 3.4 (Color online) $R_{A A}$ of $\pi^{0}$ as a function of transfer momentum in central $\mathrm{Cu}+\mathrm{Cu}$ collisions at $\sqrt{s_{N N}}=22.4 \mathrm{GeV}, 62.4 \mathrm{GeV}$ and $200 \mathrm{GeV}$ shown in black, blue and red, respectively. The shaded bands represent parton energy loss calculations [12]

\section{Summary and outlook}

The PHENIX experiment at RHIC has performed a set of measurements of light mesons $\left(\pi^{0}, \mathrm{~K}_{S}^{0}, \eta, \omega, \eta^{\prime}, \phi\right)$ via multi-particle decay channels at intermediate and high $p_{T}$ in different collision systems. A first measurement of $\eta^{\prime} \rightarrow$ $\eta \pi^{+} \pi^{-}$at RHIC performed by PHENIX at high- $p_{T}$ is reported here. It would be very interesting to measure the production rate of $\eta^{\prime}$ in $\mathrm{Au}+\mathrm{Au}$ collisions in view of predictions of a possible enhancement as its mass decreases in the hot and dense matter [16]. In $\mathrm{p}+\mathrm{p}$ and $\mathrm{d}+\mathrm{Au}$ $p_{T}$-dependent non-identical meson-to-meson ratios exhibit a universal flat behavior above $p_{T}=2.5 \mathrm{GeV} / c$ demonstrating that $p_{T}$ spectra of all measured light mesons have the same slope in this $p_{T}$ range. Cold nuclear matter does not affect the production rates of light mesons at $p_{T}>2.5 \mathrm{GeV} / c$ as seen from the matching of meson-to-meson ratios measured in $\mathrm{p}+\mathrm{p}$ and $\mathrm{d}+\mathrm{Au}$. Using the new $\mathrm{p}+\mathrm{p}$ reference, $\phi$ meson's $R_{A A}$ has been derived in central $\mathrm{Au}+\mathrm{Au}$ collisions. At intermediate- $p_{T} \phi$ meson is less suppressed than $\pi^{0}$ and $\eta$ and it exhibits similar suppression level to those of $\pi^{0}$ and $\eta$ at high- $p_{T}$. Parton energy loss calculations reproduce well the $\pi^{0}$ meson suppression for $p_{T}>3 \mathrm{GeV} / c$ in $\mathrm{Cu}+\mathrm{Cu}$ collisions at $\sqrt{s_{N N}}=62.4 \mathrm{GeV}$ and $200 \mathrm{GeV}$. The onset of jet quenching is between $22.4 \mathrm{GeV}$ and $62.4 \mathrm{GeV}$.

Acknowledgements The author acknowledges support by the Israel Science Foundation, the MINERVA Foundation and the Nella and Leon Benoziyo Center of High Energy Physics Research.

\section{References}

1. S.S. Adler et al., Phys. Rev. Lett. 91, 172301 (2003)

2. K. Adcox et al., Nucl. Instrum. Methods A 499, 469 (2003)

3. S.S. Adler et al., Phys. Rev. C 74, 024904 (2006) 
4. A. Adare et al., Phys. Rev. Lett. 98, 232301 (2007)

5. S.S. Adler et al., Phys. Rev. C 75, 051902 (2007)

6. S.S. Adler et al., Phys. Rev. C 75, 024909 (2007)

7. V. Ryabov (PHENIX Collaboration), Int. J. Mod. Phys. E 16, 1864 (2007)

8. D. Pal (PHENIX Collaboration), Nucl. Phys. A 774, 489 (2006)

9. V. Ryabov (PHENIX Collaboration), J. Phys. G 35, 044030 (2008)
10. S.S. Adler et al., Phys. Rev. Lett. 91, 241803 (2003)

11. K. Reygers (PHENIX Collaboration), arXiv:0804.4562 [nucl-ex]

12. A. Adare et al., arXiv:0801.4555 [nucl-ex]

13. I. Vitev, Phys. Lett. B 639, 38 (2006)

14. A. Adare et al., Phys. Rev. D 76, 051106 (2007)

15. A. Adare et al., arXiv:0801.4020 [nucl-ex]

16. J.I. Kapusta et al., Phys. Rev. D 53, 5028 (1996) 